\title{
Pyrolysis mass spectrometry in epidemiological and population genetic studies of Haemophilus influenzae
}

\author{
N. I. LEAVES, P. R. SISSON*, R. FREEMAN* and J. Z. JORDENS \\ Oxford Public Health Laboratory, Level 6/7, John Radcliffe Hospital, Headington, Oxford, OX3 9DU and \\ * Newcastle Public Health Laboratory. Newcastle General Hospital, Westgate Road, Newcastle Upon Tyne \\ NE4 $6 B E$
}

\begin{abstract}
Haemophilus influenzae serotype b (Hib) vaccines have reduced the amount of invasive Hib disease in immunised infants. However, Hib disease remains in unvaccinated infants and adults and non-capsulate $H$. influenzae (NCHi) still causes infections, including outbreaks of respiratory disease. Characterisation of strains and the bacterial population as a whole is therefore necessary to detect outbreaks of infection with NCHi or changes in the population, for example, to vaccine-resistant clones of Hib. The rapid, simple and objective technique of pyrolysis mass spectrometry (PMS) was investigated as an alternative to current complex, subjective methods. PMS was compared with ribotyping and multilocus enzyme electrophoresis (MLEE) for population genetic analyses of $\mathrm{Hib}$ and with ribotyping and protein profiling for epidemiological analyses of NCHi. PMS clustered all the isolates of Hib together whereas MLEE and ribotyping distinguished certain clones - this is probably because the three methods examine different (and unrelated) characteristics of the organisms. The PMS results were essentially similar to those from ribotyping and protein profiling for the epidemiological analyses of outbreaks of NCHi disease. Therefore, PMS is probably unsuitable for comparisons of Hib populations but it is a useful addition to the arsenal of techniques for the characterisation of $\mathrm{NCHi}$.
\end{abstract}

\section{Introduction}

In October 1992, Haemophilus influenzae serotype b (Hib) vaccines were introduced into the routine immunisation schedule of infants in the UK and this has led to a dramatic reduction in the incidence of $\mathrm{Hib}$ disease [1]. Therefore, it is important to monitor the population structure of $H$. influenzae in the postvaccine era to detect clones that may have a propensity to persist despite the antibodies stimulated by vaccine. Non-capsulate $H$. influenzae (NCHi) were expected to be unaffected by Hib vaccine and recently there has been an increase in the incidence of severe $\mathrm{NCHi}$ disease in the UK (M.P.E. Slack, personal communication). NCHi is now recognised as an important cause of invasive disease [2] and can cause nosocomial outbreaks of respiratory disease in susceptible individuals

Received 13 June 1996; accepted 13 Aug. 1996.

Corresponding author: Dr N. I. Leaves. Present address and address for correspondence: Wellcome Trust Centre for Human Genetics, Nuffield Orthopaedic Centre, Windmill Road, Headington, Oxford OX3 7BN.
$[3,4]$, necessitating costly infection control measures. Highly discriminatory methods of isolate characterisation are required to confirm putative outbreaks of infection.

For epidemiological studies, $H$. influenzae strains have been characterised successfully by outer-membrane protein (OMP) profiling [5], multilocus enzyme electrophoresis (MLEE) [6], ribotyping [7] and PCRbased methods [3]. These techniques are either slow, phenotypic or have poor reproducibility. MLEE has been applied extensively to the study of populations of $H$. influenzae and has shown a high degree of clonality among epidemiologically unrelated strains; however, MLEE is extremely labour intensive. A comparison of ribotyping and MLEE showed discordance between clusters of strains, indicating that MLEE remains the standard for population genetic studies [7].

In pyrolysis mass spectrometry (PMS), smears of bacterial cells are subjected to thermal degradation at $>300^{\circ} \mathrm{C}$ in the absence of oxygen and the gaseous products of pyrolysis are analysed by mass spectro- 
metry. This has been shown to be useful in epidemiological investigations of many bacterial species, including Staphylococcus aureus [8] and Pseudomonas aeruginosa [9]. The advantages of PMS are its simplicity, speed and objective analysis. PMS uses data from the whole organism and hence may prove a more appropriate test for clustering analyses than other techniques, e.g., ribotyping, which examine a tiny proportion of the bacterial genome.

The aim of the present study was to compare PMS with previously used methods of characterising $H$. influenzae in epidemiological and population genetic studies. PMS was applied to the analysis of three collections of $H$. influenzae. Two collections of isolates were from putative outbreaks of infection with $\mathrm{NCHi}$ that had been characterised previously by OMP profiling and ribotyping [3]; these outbreaks both occurred in 1991, i.e., before the introduction of Hib vaccine. The third collection of strains consisted of Hib that had been characterised previously by MLEE and ribotyping [7].

\section{Materials and methods}

\section{Bacterial strains}

Two collections of NCHi from putative outbreaks of respiratory disease at hospitals $\mathrm{A}$ and $\mathrm{B}$ were received by the Haemophilus Reference Laboratory (HRL) at Oxford. The isolates from hospital A were from four residents of a nursing home. Thirteen isolates were received from hospital $\mathrm{B}$; seven isolates were associated with an outbreak of respiratory infection and six were from the same hospital but were thought to be unrelated to the outbreak. Epidemiologically unrelated but phenotypically similar controls were selected from the HRL collection of strains and were included in the subsequent analysis for comparison [3]. Hib strains of known MLEE type were kindly provided by Professor E. R. Moxon [6, 7]. In addition, two Hib isolates from ancestral division II were included in the analysis; these were the OMP control subtypes BK-8H and BK$17 \mathrm{H}$ [7]. All isolates had previously been typed by protein profiles, ribotyping with EcoRI restriction and a cDNA probe, and multilocus enzyme electrophoresis (MLEE) assaying 17 enzymes $[3,6,7]$. The OMP subtypes of the Hib strains were not used, as OMPs have been shown previously to be poorly discriminatory for Hib [7].

\section{$P M S$}

PMS was performed as described previously [9]. Briefly, bacterial growth was smeared on to pyrolysis foils which were inserted into pyrolysis tubes and dried. Samples were then pyrolysed in single batches on a Horizon Instruments PYMS 200X pyrolysis mass spectrometer at $530^{\circ} \mathrm{C}$ induced for $4 \mathrm{~s}$. The gaseous products of pyrolysis were ionised by a crossing beam of electrons and the ion intensities for mass:charge ratios 50 to 200 were analysed. Isolates were coded, analysed in triplicate and the whole experiment was duplicated so that each bacterial isolate was analysed six times. The spectral data were subjected to multivariate analysis as summarised elsewhere [9].

\section{Results}

\section{Non-capsulate $H$. influenzae}

The protein profiling and ribotyping results for isolates from the two putative outbreaks of $\mathrm{NCHi}$ disease have been reported previously [3]. The ordination diagrams produced by PMS and subsequent data analysis showed that the outbreak-associated isolates from hospital A were clustered and were readily distinguished from the control isolates. However, PMS showed that one of the four outbreak-associated isolates from hospital A was distinguished from the other three (with 95\% confidence); this had not been observed with any other method of characterisation. The results from the three methods for the isolates from hospital $B$ were concordant; the isolates from hospital B could not be resolved easily from one of the epidemiologically unrelated controls. The results for protein profiling, ribotyping and PMS analysis for the isolates from hospitals A and B are summarised in Table 1.

\section{H. influenzae serotype $b$}

The MLEE data and ribotypes have been previously reported [7] and are shown together with the data from PMS in Table 2. The three data sets were discordant; isolates were readily distinguished by ribotyping and MLEE but PMS did not distinguish any isolate (at $95 \%$ confidence).

\section{Discussion}

Epidemiological and population genetic analyses of $H$. influenzae require easily performed, rapid, genotypic and comparative methods. The present study showed that for epidemiological analyses of outbreaks of infection with $\mathrm{NCHi}$, PMS, ribotyping and OMP profiling produced essentially equivalent results with the exception of one outbreak-associated isolate from hospital A which was distinguished only by PMS. In addition, PMS confirmed the problems associated with characterising NCHi biotype II, ampicillin- and trimethoprim-resistant strains because of the clonality within this phenotype $[3,10]$. These data suggest that PMS is extremely discriminatory for $\mathrm{NCHi}$ and probably suitable for epidemiological studies; however, it is important to note that epidemiological conclusions drawn from the use of this technique alone may be discordant with those based on other techniques. This reinforces the fact that epidemiological conclusions 
Table 1. Assays of non-capsulate $H$. influenzae

\begin{tabular}{clccc}
\hline $\begin{array}{l}\text { Outbreak } \\
\text { and isolate }\end{array}$ & Source & $\begin{array}{c}\text { OMP } \\
\text { profile }\end{array}$ & $\begin{array}{c}\text { Ribotyping } \\
\text { pattern* }\end{array}$ & $\begin{array}{c}\text { PMS } \\
\text { cluster }\end{array}$ \\
\hline $\begin{array}{c}\text { Hospital A } \\
1\end{array}$ & $\begin{array}{l}\text { Unrelated hospital } \\
\text { Unrelated hospital }\end{array}$ & 1 & 1 & 1 \\
2 & Unrelated hospital & 2 & 2 & 2 \\
3 & Hospital A outbreak? & 4 & 3 & 3 \\
4 & Hospital A outbreak? & 4 & 4 & 4 \\
5 & Hospital A outbreak? & 4 & 4 & 5 \\
6 & Hospital A outbreak? & 4 & 4 & 4 \\
7 & Hospital B outbreak? & 1 & 4 & 4 \\
Hospital B & Hospital B outbreak? & 1 & 1 & 1 \\
1 & Hospital B outbreak? & 1 & 1 & 1 \\
2 & Hospital B outbreak? & 1 & 1 & 1 \\
3 & Hospital B outbreak? & 1 & 1 & 1 \\
4 & Hospital B outbreak? & 1 & 1 & 1 \\
5 & Hospital B, other & 2 & 1 & 1 \\
6 & Hospital B, other & 3 & 2 & 2 \\
7 & Hospital B, other & 4 & 3 & 3 \\
8 & Hospital B, other & 5 & 4 & 4 \\
9 & Hospital B, other & 6 & 5 & 5 \\
10 & Hospital B outbreak? & NT & 6 & 6 \\
11 & Unrelated hospital & 1 & 1 & 1 \\
12 & Unrelated hospital & 1 & 7 & 1 \\
13 & Unrelated hospital & NT & $1 \mathrm{~b}$ & 1 \\
15 & Unrelated hospital & & 1 \\
\hline
\end{tabular}

OMP, outer-membrane protein; * Ribotyping patterns $1 \mathrm{a}$ and $1 \mathrm{~b}$ differed from pattern 1 by one band. PMS = pyrolysis mass spectrometry; NT, not tested.

Table 2. Analysis of $H$. influenzae serotype $\mathrm{b}$

\begin{tabular}{lccl}
\hline $\begin{array}{l}\text { Strain reference } \\
\text { no. }\end{array}$ & $\begin{array}{c}\text { MLEE† } \\
\text { electrotype no. }\end{array}$ & Ribotype $\$$ & PMS \\
\hline RM7118 & 1.7 & 6 & Clustered \\
RM7430 & 8 & 19 & Clustered \\
RM6107 & 31 & 3 & Clustered \\
RM7020 & 50 & 3 & Clustered \\
RM7017 & 52 & 8 & Clustered \\
RM7109 & 21.9 & 18 & Clustered \\
RM7419 & 60 & 8 & Clustered \\
RM6094 & 71 & 2 & Clustered \\
RM1005 & 92 & 1 & Clustered \\
RM8012 & 122 & 7 & Clustered \\
RM8069 & 133 & 6 & Clustered \\
RM7414 & 140 & 6 & Clustered \\
NL BK-8H & Unknown & 20 & Clustered \\
NL BK-17H & Unknown & 22 & Clustered \\
\hline
\end{tabular}

* Strain reference number from Musser et al. [6].

$\dagger$ MLEE, multilocus enzyme electrophoresis electrotype number from Musser et al. [6].

Ribotyping nomenclature of Leaves and Jordens [7].

§Clustered at $95 \%$ confidence.

should be based upon the use of multiple techniques [11].

The introduction of Hib vaccine in the UK has increased the importance of monitoring Hib populations because it is possible that a clone might emerge that is able to withstand antibodies stimulated by the vaccine. Previously, OMP subtyping, ribotyping and MLEE have been used to compare Hib populations; however, the results were discordant and MLEE is still regarded as the reference tool for population genetic studies of Hib [7]. In the present study of Hib, PMS failed to distinguish any Hib isolate, including isolates from different major phylogenetic lineages (ancestral divisions I and II), effectively clustering them all. These data are discordant with ribotypes and MLEE as used here, probably because PMS analyses an entire bacterial cell whereas MLEE and ribotyping analyse less of the organism. Indeed, the lack of heterogeneity detected by PMS is probably typical of $\mathrm{Hib}$ and, therefore, may be more representative than either MLEE or ribotyping, in which minute polymorphisms are detected in just a few genes and hence might bias calculations of similarity between whole genomes.

PMS has been shown to be a useful technique in epidemiological analyses of NCHi. The use of PMS in population genetic studies remains uncertain, although we speculate that the technique might prove suitable for this purpose. The applicability of PMS in population genetics will probably be clarified only by direct comparisons between large areas of DNA sequence from strains and comparison of similarity results based on these with those from MLEE, ribotyping and PMS.

We thank all those who have submitted bacterial isolates to the Haemophilus Reference Laboratory at Oxford. We also thank Professor E. R. Moxon for the kind gift of bacterial strains of known MLEE subtype and E. C. Anderson for biotyping and serotyping data.

\section{References}

1. Teare EL, Fairley CK, White J, Begg NT. Efficacy of Hib vaccine. Lancet 1994; 344: 828-829.

2. Jordens JZ, Slack MPE. Haemophilus influenzae: Then and 
Now. Eur J Clin Microbiol Infect Dis 1995; 14: 935-948.

3. Jordens JZ, Leaves NI, Anderson EC, Slack MPE. Polymerase chain reaction-based characterization of non-capsulate Haemophilus influenzae. J Clin Microbiol 1993; 31: 2981-2987.

4. Sturm AW, Mostert R, Rouing PJE, van Klingeren B, van Alphen L. Outbreak of multiresistant non-encapsulated Haemophilus influenzae infections in a pulmonary rehabilitation centre. Lancet 1990; 335: 214-216.

5. van Alphen L, Riemans T, Poolman J, Zanen HC. Characteristics of major outer membrane proteins of Haemophilus influenzae. $J$ Bacteriol 1983; 155: 878-885.

6. Musser JM, Kroll JS, Granoff DM et al. Global genetic structure and molecular epidemiology of encapsulated Haemophilus influenzae. Rev Infect Dis 1990; 12: 75-111.

7. Leaves NI, Jordens JZ. Development of a ribotyping scheme for Haemophilus influenzae type b. Eur J Clin Microbiol Infect
Dis 1994; 13: 1038-1045.

8. Gould FK, Freeman R, Sisson PR, Cookson BD, Lightfoot NF. Inter-strain comparison by pyrolysis mass spectrometry in the investigation of Staphylococcus aureus nosocomial infection. J Hosp Infect 1991; 19: 41-48.

9. Sisson PR, Freeman R, Gould FK, Lightfoot NF. Strain differentiation of nosocomial isolates of Pseudomonas aeruginosa by pyrolysis mass spectrometry. $J$ Hosp Infect 1991; 19: $137-140$.

10. Leaves NI, Jordens JZ. Analysis of the prevaccine population of noncapsulate Haemophilus influenzae and identification of a putative epidemic clone. Eur J Clin Microbiol Infect Dis 1996; 15: $410-414$.

11. Maslow JN, Mulligan ME, Arbeit RD. Molecular epidemiology: application of contemporary techniques to the typing of microorganisms. Clin Infect Dis 1993; 17: 153-164. 